Cornelia C. Bergmann, PhD

Department of Neuroscience, Cleveland Clinic

Lerner Research Institute;

Professor, Cleveland Clinic Lerner College

of Medicine of Case Western Reserve University,

Cleveland, $\mathrm{OH}$
Robert H. Silverman, PhD

Department of Cancer Biology, Cleveland Clinic

Lerner Research Institute; Professor, Cleveland

Clinic Lerner College of Medicine of Case Western

Reserve University, Cleveland, $\mathrm{OH}$

\title{
COVID-19: Coronavirus replication, pathogenesis, and therapeutic strategies
}

$\mathrm{H}$ UMAN CORONAVIRUSES, along with influenza virus, human metapneumovirus, respiratory syncytial virus, and rhinovirus, are endemic and cause approximately $15 \%$ to $30 \%$ of annual respiratory tract infections. Coronavirus infections are generally mild in healthy adults, obviating any urgent need to develop treatments or vaccines. However, outbreaks of acute respiratory distress syndrome (ARDS) due to novel, highly pathogenic strains-severe acute respiratory syndrome coronavirus (SARS-CoV), Middle East respiratory syndrome coronavirus (MERS-CoV), and now, SARS-CoV-2-have revealed the potency and danger of this expanding family of pathogens that have the capacity to kill many thousands of people around the world if not geographically contained. ${ }^{1}$

As in severe SARS and MERS disease, the mortality rate is disproportionately high in the elderly and patients with preexisting comorbidities such as heart disease, diabetes mellitus, hypertension, and renal disease. ${ }^{2}$ Higher morbidity in the elderly may partly be attributed to muted interferon antiviral responses (although the suggestive study has not yet been peer-reviewed $)^{3}$ as well as overall lower adaptive immunity, ${ }^{2}$ resulting, paradoxically, in longer courses of hyperactivity of the innate immune system ("cytokine storm").

\section{A ZOONOTIC INFECTION}

Bats have been implicated as the likely source of SARS-CoV-2, as both SARS-CoV and MERS-CoV are genetically similar to viruses recovered from bats, and bat coronaviruses can use human receptors for cell entry. ${ }^{4}$ How-

Disclosure: Dr. Silverman is a Scientific Advisory Board member of Sator Therapeutics, LLC (Cleveland).

doi:10.3949/ccjm.87a.20047 ever, phylogenetic studies, looking at sequencebased virus evolution, suggest that the virus is not transmitted directly from bats to humans but rather first infects intermediate animal hosts in close contact with humans. In the case of SARS-CoV, these can be civets or raccoon dogs sold at crowded markets; for MERS-CoV, they can be domesticated dromedary camels. ${ }^{4}$

Transmission from bats to intermediate hosts and then to humans, as well as from human to human, all involve viral adaptation, slight changes in viral sequence to improve fitness in the new host. This is not unique to coronaviruses, as endemics and pandemics also occur when novel influenza $A$ virus strains emerge in the human population from an animal host. ${ }^{5}$ Similar to introduction of Ebolavirus and human immunodeficiency virus 1 by mammals, many other viruses circulating in wild animals have the potential for zoonotic transmission. ${ }^{6}$

SARS-CoV-2, the causative agent for the pandemic corona virus disease of 2019 (COVID-19) outbreak, was first found in Wuhan, China, and initial analysis of viral RNA obtained from patients hospitalized in late 2019 revealed it was $96 \%$ identical at the whole-genome level to a bat SARS-like coronavirus.

Uniquely, SARS-CoV-2 can be transmitted by people who are infected but have no symptoms, not just by symptomatic patients. Concern about potential spread of SARSCoV-2 to household cats has emerged from a news report of infection in a tiger in the Bronx Zoo. Ferrets can be infected, with intraspecies transmission, ${ }^{8}$ and cats can also be infected and transmit the virus to other cats, while dogs have low susceptibility. However, it is unknown if any of these animals can transmit the virus to humans. ${ }^{9}$

\section{Understanding of the virus and how it causes disease points the way to potential treatments}




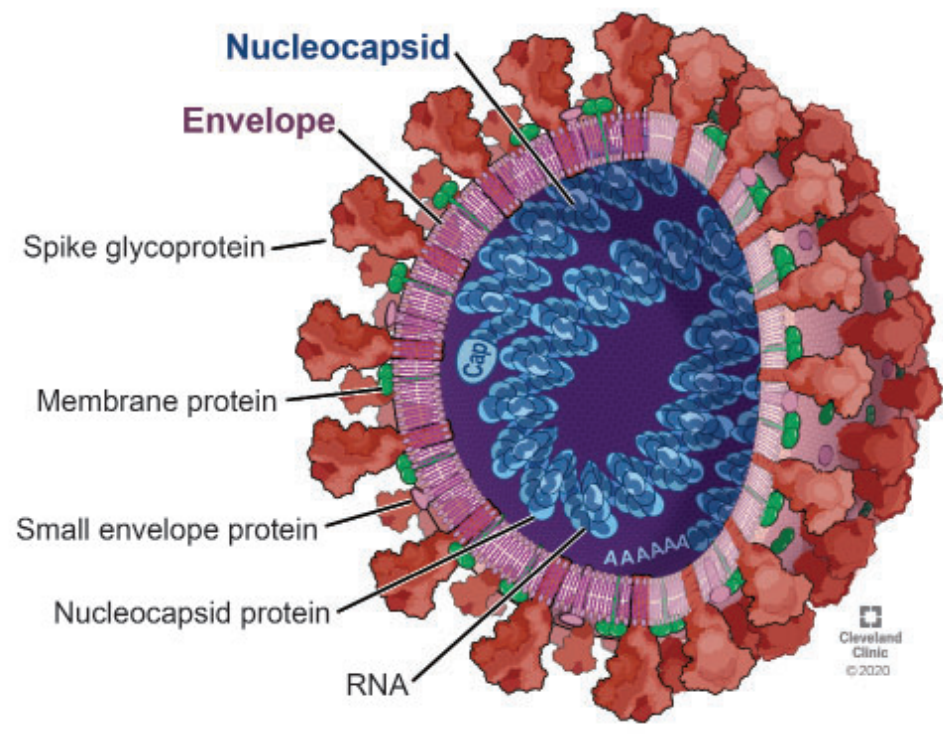

Figure 1. Structure of coronaviruses.

\section{STRUCTURE AND GENOME} OF CORONAVIRUSES

Coronaviruses are spherical enveloped viruses containing a single strand of positivesense RNA (similar to host mRNA) of approximately 26 to $32 \mathrm{~kb}^{10}$ Their defining morphologic features are club-shaped projec-

Only a few changes are needed to adapt an animal virus to humans tions from the viral envelope resembling a crown or a solar corona and made of a highly glycosylated protein named spike protein. Their other 3 structural proteins are the envelope, membrane, and nucleocapsid proteins (Figure 1).

The first two-thirds of the genome consists of 2 large overlapping open reading frames that encode 16 nonstructural proteins, including proteases, RNA-dependent RNA polymerase ( $p r R d R p)$, RNA helicase, primase, and others, that form the viral replicase complex, a platform to propagate viral mRNAs. These nonstructural proteins are all potential targets for therapies, which would in theory work against all coronaviruses (Figure 2). ${ }^{1,8,10-15}$

The remaining portion of the genome includes interspersed open reading frames for the structural proteins, as well as a number of accessory proteins generally nonessential for replication in tissue culture but capable of suppressing immune responses and enhancing pathogenesis. ${ }^{10,16}$

\section{HOW THE VIRUS GETS IN}

Features of coronavirus transmission, replication, and pathogenesis are determined by both the viral genome and the human host.

Coronavirus spike proteins are key determinants for virus attachment and entry into target cells. The receptor for both SARS-CoV and SARS-CoV-2 is angiotensin-converting enzyme 2 (ACE2), ${ }^{11,12}$ a cell-surface enzyme contributing to control of blood pressure. SARS-CoV cell entry is independent of ACE2 catalytic activity.

Entry involves 2 spike protein subunits, which mediate distinct functions. The S1 subunit mediates ACE2 attachment through the receptor-binding domain. The S2 subunit, containing the fusion peptide and transmembrane domains, drives fusion of viral and host cell membranes. To be activated for fusion, the spike protein must be cleaved at 2 sites directly at the cell membrane, through endosomes, or both. The sequence of the cleavage sites, one located at the border of S1 and S2 subunits, the other (S2') within S2 just upstream of the fusion peptide, provide substrates for a variety of cellular proteases and determine cleavage efficiency.

The route or routes of infection thus depend on the proteases available in different cell types and the protease cleavage sites. ${ }^{17}$ This is also demonstrated by involvement of the cellular serine protease TMPRSS2 (transmembrane protein serine protease 2 ) and activities of furin and endosomal cathepsins B and $\mathrm{L}$ in SARS-CoV-2 entry. ${ }^{11}$ TMPRSS2 activity is also involved in viral spread and pathogenesis in SARS-CoV-infected and MERS-CoV-infected mouse models. ${ }^{18}$

Host proteases that cleave the $S$ protein are also potential targets for antiviral drugs. A higher rate of SARS-CoV-2 infections compared with SARS-CoV infections may be at least partially explained by a higher affinity of spike protein for ACE2. ${ }^{12}$ The sequence divergence in both the receptorbinding domain and cleavage domains in the spike protein between SARS-CoV-2 and the bat virus highlight how only a few changes are needed to adapt an animal virus to humans. ${ }^{7,11,12,19}$ 


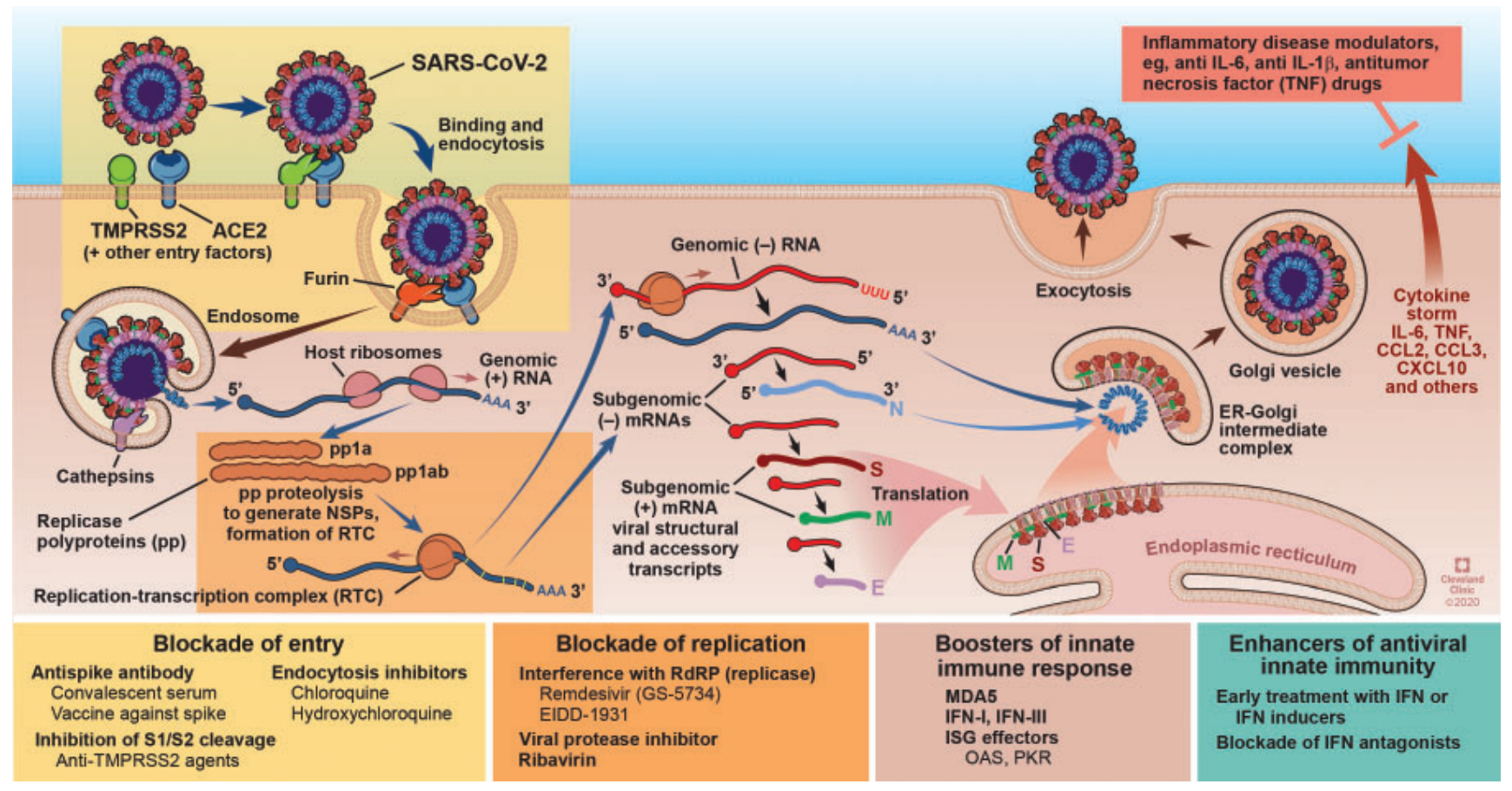

Figure 2. Overview of COVID-19, SARS-CoV-2 replication, and therapeutic targets.

Upper left. Virus entry entails binding the angiotensin-converting enzyme 2 (ACE2) receptor and cleavage by the serine protease TMPRSS2 (in green) to allow fusion with the host membrane. Other cellular proteases, eg, furin (in orange), facilitate $\mathrm{pH}$-dependent entry through the endocytic pathway. The predominant entry routes are cell type-specific and dependent on availability of select proteases.

Middle. Following uncoating and release of viral RNA into the cytoplasm, translation of open reading frame 1a (ORF1a) and ORF1ab produces the polyproteins pp1a and pp1ab. These in turn are processed by viral proteases (encoded by ORF1a) to yield 16 nonstructural proteins. Formation of the RNA replicase-transcriptase complex (RTC) uses rough endoplasmic reticulum (ER)-derived membranes. The RTC drives synthesis of (-)RNAs. Full-length (-)RNA copies of the genome provide templates for full-length (+)RNA genomes. Transcription further produces a subset of subgenomic RNAs, including those encoding all structural and accessory proteins.

Right. The translated structural proteins and genomic RNA are assembled into the viral nucleocapsid and envelope in the ER-Golgi intermediate compartment, and are subsequently released by exocytosis.

Bottom. Potential strategies for treatment. Anti-TMPRSS2 or chloroquine treatment in experimental animals will reveal efficacy of targeting select proteases or entry pathways in limiting infection, while simultaneously monitoring effects on innate and adaptive immunity. The replication cycle can be blocked at several stages using single or combined treatment paradigms: virus entry can be inhibited by antispike antibodies elicited by vaccines to block attachment or by preventing fusion using relevant protease inhibitors. ${ }^{11}$ RTC formation and transcription-replication events can be targeted using viral protease inhibitors or nucleoside analogues (GS-5734 or EIDD-1931). ${ }^{15}$ Interferon (IFN) responsiveness can be increased by early exogenous IFN treatment, ${ }^{13}$ IFN inducer treatment, repression of viral IFN antagonists, and enhancement of host antiviral IFN pathways. The "cytokine storm" induced as a host response to rampant virus replication may be targeted by administration of select anti-inflammatory immune modulators, which are already given to patients with inflammatory disorders. Drugs targeting viral replication may also be combined with treatments that control detrimental immune responses. The ferret model will provide a useful tool to test multiple therapeutic and preventive treatments. ${ }^{8}$ 
$\square$ THE BODY MOUNTS AN INNATE IMMUNE RESPONSE

Interferons I and III are cytokines with critical roles in the innate immune response against viral infections. ${ }^{20}$ Virus-infected cells induce and secrete interferon I molecules that bind to the cell surface receptor IFNAR (interferon III uses a different receptor), thereby triggering the Jak-Stat (Janus kinase/signal transducer and activator of transcription) signaling pathway that switches on many antiviral genes. The interferon-stimulated genes are then transcribed into RNA and translated into proteins that suppress viral replication and spread.

\section{HOW THE VIRUS EVADES THE HOST RESPONSE}

During coevolution with their hosts, viruses have learned to counteract the interferon antiviral response. Like other human coronaviruses, SARS-CoV-2 can at least partly evade innate immunity to gain a foothold in humans, a critically important step in the infection cycle. Although mechanistic insights are as yet unavailable, we do have a good understanding of how other coronaviruses evade During interferon's antiviral activity, ${ }^{21}$ and also how coevolution with their hosts, viruses have learned to counteract the interferon antiviral response we could engage antiviral factors to promote interferon activity. ${ }^{22}$

In general, coronaviruses can potently antagonize antiviral innate immunity by interfering with both interferon production and the cellular antiviral response. ${ }^{23}$ For instance, mouse coronaviruses and MERS-CoV have accessory proteins that block an interferon response pathway that degrades the viral RNA (by oligoadenylate synthetase and ribonuclease $\mathrm{L}) .{ }^{24,25}$

The large number of host antiviral mechanisms and distinct viral antagonism at different steps in the virus replication cycle have made it difficult to identify the most relevant ones. Not only does each type of coronavirus encode different accessory proteins responsible for allowing the virus to escape cellular innate immune mechanisms, but distinct cell types may respond differently.

While we are only just beginning to understand the functions of the SARS-CoV-2 accessory proteins, it is clear that there are similarities and differences between the acces- sory proteins of SARS-CoV-2 and those of its closest human pathogen relative, SARS-CoV. A better understanding of the precise functions of the SARS-CoV-2 accessory proteins, especially their interaction with innate immune pathways, could lead to novel antiviral drugs that promote the innate immune response. The finding (not yet peer-reviewed) ${ }^{26}$ that SARS-CoV-2 was more sensitive to interferon than SARS-CoV raises hope that giving interferon or interferon inducers very early in the infection could be beneficial, and, perhaps, less likely to cause harm than using interferon later in COVID-19. ${ }^{13}$

\section{WHY DO SOME PEOPLE GET SO SICK, BUT OTHERS ARE FINE?}

One of the most problematic features of SARS$\mathrm{CoV}-2$ infection is the broad spectrum of disease, ranging from no symptoms to mild flulike symptoms, anosmia, fever, nonproductive cough, dyspnea, and fatigue to acute respiratory distress syndrome, the main cause of death. While multiple organs, including the heart, kidneys, liver, and gastrointestinal tract, are injured, it remains to be resolved to what extent tissues are damaged by infection, hypoxia, or the immune response. Complications may also involve the central nervous system, either by direct infection or secondary damage. ${ }^{27,28}$

Different ACE2 expression? ACE2 is expressed in various cell types of the lung, including alveolar epithelial cells, pneumocytes, and bronchial transient secretory cells, as well as enterocytes of the small intestine, heart (pericytes), and kidney. These are the same tissues that the virus affects, but studies with SARS-CoV indicate that ACE2 expression is not the only determinant of susceptibility. ${ }^{29-32}$ More research is needed to assess to what extent ACE2 surface expression or polymorphisms, or other coreceptors and proteoglycan moieties, are markers of tissue susceptibility.

Renin-angiotensin system dysregulation? The finding that ACE2 is a primary SARS$\mathrm{CoV}-2$ receptor has further led to extensive discussion of dysregulation of the renin-angiotensin system, which regulates blood pressure and electrolyte balance. ${ }^{33-35}$ Conversion of angiotensin I to angiotensin II by angiotensinconverting enzyme (ACE) activates pathways 
that lead to inflammation, vasoconstriction, oxidation, and fibrosis. ACE2 activity counterbalances this pathway by cleaving both angiotensin I and angiotensin II to shorter peptides, which use distinct receptors to promote vasodilation, as well as anti-inflammatory, antioxidant, and antifibrosis activity.

ACE inhibitors and angiotensin II receptor blockers are thus commonly used in patients with cardiovascular diseases, hypertension, and diabetes, promoting the protective effects of ACE2. However, increased expression of ACE2 by use of antihypertensive drugs in animal models raised concerns of higher virus infection risk for patients receiving these drugs. Nevertheless, current recommendations are to continue treatment. ${ }^{34,35}$ The complexity of the renin-angiotensin system will require more extensive retrospective analysis of larger and ethnically diverse patient groups.

Protective host features? The apparently large percentage of infections that are asymptomatic is unique to SARS-CoV-2, but many of the pathogenic features resemble those observed in SARS and MERS-CoV infections. The protective host features underlying the asymptomatic infections are currently unknown, as testing within many countries is limited to people presenting with symptoms such as severe shortness of breath, coughing, and fever. Retrospective studies incorporating serum antibody testing and health status will provide much-needed insights.

Cytokine storm? Severe disease is associated with lymphopenia and an uncontrolled systemic inflammatory response called a cytokine storm, which ultimately leads to multiple organ failure and death. ${ }^{36,37}$ Autopsy results reveal severe damage to endothelial tissue, vasculitis-like manifestations, and atrophy of secondary lymphoid tissues. ${ }^{37}$ Early studies in COVID-19 patients showed higher plasma levels of interleukin 2 (IL-2), IL-7, granulocyte colony-stimulating factor, C-X-C motif chemokine 10, monocyte chemoattractant protein 1 , macrophage inflammatory protein 1a (chemokine [C-C motif] ligand 2), and tumor necrosis factor (TNF), but also antiinflammatory IL-10, higher in intensive care patients than in nonintensive care patients. ${ }^{38}$ Several reports also confirm high levels of IL-6 in severely ill patients. ${ }^{2,39}$ Retrospective clinical investigation of more patient cohorts without or with preexisting conditions and of those being treated with distinct anti-inflammatory immune modulators-eg, anti-TNF, anti-IL-6, anti-IL12/IL23, or anti-IL-1 betafor immune-mediated inflammatory conditions will provide much-needed guidance on treatment to stem severe COVID-19.

\section{ONCE YOU GET IT, ARE YOU IMMUNE FOR LIFE?}

Another critical unresolved aspect of COVID-19 is the establishment of adaptive immunity. Lessons from the SARS-CoV epidemic indicate that CD4 and CD8 T-cell memory lasts for up to 11 years in recovered individuals. ${ }^{40-42}$ A study of a limited number of patients hospitalized with mild or severe COVID-19 revealed humoral immunoglobulin $\mathrm{M}$ ( $\operatorname{IgM}$ ) and immunoglobulin $\mathrm{G}$ ( $\mathrm{IgG}$ ) serum responses to the viral nucleocapsid and spike proteins emerging at 10 days after symptom onset, with serconversion in a sizable majority of patients by 3 weeks. $2,43,44$ Moreover, the IgG levels correlated with virus neutralization titers.

Transfusion of convalescent plasma from recovered patients had beneficial outcomes in a small number of SARS and COVID-19 cases. ${ }^{45}$ Based on preliminary results of convalescent serum as well as in vitro and in vivo neutralization studies, clinical trials will be launched to evaluate the efficacy of spike protein-based vaccines.

A concern is the mutation rate of the virus as it spreads through the population. Viral genomes are being analyzed throughout the world and compiled in large, publicly available databases, which collate sequenced isolates and look at relationships (https:// nextstrain.org/ncov/global). Although such databases currently reflect a population naïve to the virus, similar studies can be conducted once vaccines become available to test the effects of immune pressure on the virus.

\section{VIRAL AND HOST TARGETS FOR THERAPIES AND VACCINES}

There are at least 4 potential therapeutic strategies against COVID-19, apart from supportive and oxygenation therapies such as use of ventilators:

\section{There are at least 4 potential therapeutic strategies against COVID-19}


- Direct antiviral drugs against SARSCoV-2 (eg, remdesivir)

- Indirect antiviral agents (eg, interferon I, interferon inducers, and drugs that target host proteins required for infections)

- Convalescent plasma that contains antibodies against SARS-CoV-2

- Drugs that tamp down the pathogenic hyperactive inflammatory response and cytokine storm later in disease progression.

However, we would like to emphasize that at present, these strategies are investigational only, including the off-label use of existing drugs, and may prove to show no efficacy and could be harmful in controlled clinical trials.

The emergence of 3 highly pathogenic human coronaviruses within the past 20 years predicts that more of them will continue to come along. As the timing is unpredictable, monitoring and transparent reporting of local outbreaks is imperative for early intervention.

With respect to currently circulating SARS-CoV-2 and limited overall testing, it is also unknown whether the virus is affected by seasonal changes. While physical distancing is an effective control measure to limit acute infection rates, asymptomatic carriers will likely continue to spread the virus, leading to ongoing hotspots of symptomatic infection.

A major factor influencing the future of COVID-19 is the ability of recovered people to develop protective immunity. However, the ongoing yearly infection rates by historically circulating coronaviruses, ${ }^{46}$ as well as evidence for already distinct SARS-CoV-2 variants ${ }^{47}$ suggest that established immunity may be insufficient to avoid recurring infections.

Clinical trials with drugs targeting viral proteins will reveal tolerance of the SARSCoV-2 to selective pressure and guide in development of strategies that target host proteins required for replication. ${ }^{48}$ Efficacy of vaccination strategies to elicit protective antibodies may further uncover the potential need for seasonal vaccines like those for circulating influenza viruses.

Acknowledgments: We wish to thank Drs. Mark Cameron (Case Western Reserve University) and Susan R. Weiss (University of Pennsylvania) for critical reading of the manuscript. We also thank David Schumick, Center for Medical Art and Photography, Cleveland Clinic, for expert artwork. We wish to acknowledge support by the National Institute of Allergy and Infectious Diseases of the National Institutes of Health under Awards R01NS110700, R01NS091183, R01NS086299 (to C.C.B.) and R01Al104887 and R01Al135922 (to R.H.S.).

\section{REFERENCES}

1. de Wit E, van Doremalen N, Falzarano D, Munster VJ. SARS and MERS: recent insights into emerging coronaviruses. Nat Rev Microbiol 2016; 14(8):523-534. doi:10.1038/nrmicro.2016.81

2. Chen Y, Li L. SARS-CoV-2: virus dynamics and host response. Lancet Infect Dis 2020 Mar 23;S1473-3099(20)30235-8. doi:10.1016/S1473-3099(20)30235-8

3. Blanco-Melo D, Nilsson-Payant BE, Liu W-C, et al. SARS-CoV-2 launches a unique transcriptional signature from in vitro, ex vivo, and in vivo systems. bioRxiv 2020. doi:10.1101/2020.03.24.004655

4. Cui J, Li F, Shi Z-L. Origin and evolution of pathogenic coronaviruses. Nat Rev Microbiol 2019; 17(3):181-192. doi:10.1038/s41579-018-0118-9

5. Paules C, Subbarao K. Influenza. Lancet 2017; 390(10095):697-708. doi:10.1016/S0140-6736(17)30129-0

6. Olival KJ, Hosseini PR, Zambrana-Torrelio C, Ross N, Bogich TL, Daszak P. Host and viral traits predict zoonotic spillover from mammals. Nature 2017; 546(7660):646-650. doi:10.1038/nature22975

7. Zhou P, Yang X-L, Wang X-G, et al. A pneumonia outbreak associated with a new coronavirus of probable bat origin. Nature 2020; 579(7798):270-273. doi:10.1038/s41586-020-2012-7

8. Kim YI, Kim S-G, Kim S-M, et al. Infection and rapid transmission of SARS-CoV-2 in ferrets. Cell Host Microbe 2020 Apr 5; S19313128(20)30187-6. doi:10.1016/j.chom.2020.03.023

9. Shi J, Wen Z, Zhong G, et al. Susceptibility of ferrets, cats, dogs, and other domesticated animals to SARS-coronavirus 2. Science $2020 \mathrm{Apr}$ 8;eabb7015. doi:10.1126/science.abb7015

10. Fehr AR, Perlman S. Coronaviruses: an overview of their replication and pathogenesis. Methods Mol Biol 2015; 1282:1-23. doi:10.1007/978-1-4939-2438-7_1

11. Hoffmann M, Kleine-Weber H, Schroeder S, et al. SARS-CoV-2 cell entry depends on ACE2 and TMPRSS2 and Is blocked by a clinically proven protease inhibitor. Cell 2020; 181(2):271-280.e8. doi:10.1016/j.cell.2020.02.052

12. Lan J, Ge J, Yu J, et al. Structure of the SARS-CoV-2 spike receptorbinding domain bound to the ACE2 receptor. Nature 2020 Mar 30. doi:10.1038/s41586-020-2180-5

13. Sallard E, Lescure F-X, Yazdanpanah Y, Mentre F, Peiffer-Smadja N. Type 1 interferons as a potential treatment against COVID-19. Antiviral Res 2020 Apr 7:178:104791. doi:10.1016/j.antiviral.2020.104791

14. Totura AL, Bavari S. Broad-spectrum coronavirus antiviral drug discovery. Expert Opin Drug Discov 2019; 14(4):397-412. doi:10.1080/17460441.2019.1581171

15. Sheahan TP, Sims AC, Zhou S, et al. An orally bioavailable broadspectrum antiviral inhibits SARS-CoV-2 in human airway epithelial cell cultures and multiple coronaviruses in mice. Sci Transl Med 2020; Apr 6;eabb5883. doi:10.1126/scitranslmed.abb5883

16. Lu R, Zhao X, Li J, et al. Genomic characterisation and epidemiology of 2019 novel coronavirus: implications for virus origins and receptor binding. Lancet 2020; 395(10224):565-574. doi:10.1016/S0140-6736(20)30251-8

17. Millet JK, Whittaker GR. Host cell proteases: critical determinants of coronavirus tropism and pathogenesis. Virus Res 2015; 202:120-134. doi:10.1016/j.virusres.2014.11.021

18. Iwata-Yoshikawa N, Okamura T, Shimizu Y, Hasegawa H, Takeda M, Nagata N. TMPRSS2 contributes to virus spread and immunopathology in the airways of murine models after coronavirus infection. J Virol 2019; 93(6):1815-1818. doi:10.1128/JVI.01815-18

19. Zhang $T$, Wu Q, Zhang Z. Probable pangolin origin of SARS-CoV-2 associated with the COVID-19 outbreak. Curr Biol 2020; 30(7):13461351.e2. doi:10.1016/j.cub.2020.03.022

20. Borden EC, Sen GC, Uze G, et al. Interferons at age 50: past, current and future impact on biomedicine. Nat Rev Drug Discov 2007; 6(12):975-990. doi:10.1038/nrd2422 
21. Weiss SR. Forty years with coronaviruses. J Exp Med 2020; 217(5):e20200537. doi:10.1084/jem.20200537

22. Butchi NB, Hinton DR, Stohlman SA, et al. Ifit2 deficiency results in uncontrolled neurotropic coronavirus replication and enhanced encephalitis via impaired alpha/beta interferon induction in macrophages. J Virol 2014; 88(2):1051-1064. doi:10.1128/JVI.02272-13

23. Kindler E, Thiel V. SARS-CoV and IFN: too little, too late. Cell Host Microbe 2016; 19(2):139-141. doi:10.1016/j.chom.2016.01.012

24. Zhao L, Birdwell LD, Wu A, et al. Cell-type-specific activation of the oligoadenylate synthetase-RNase $L$ pathway by a murine coronavirus. J Virol 2013; 87(15):8408-8418. doi:10.1128/JVI.00769-13

25. Thornbrough JM, Jha BK, Young B, et al. Middle East respiratory syndrome coronavirus NS4b protein inhibits host RNase $L$ activation. mBio 2016; 7(2):e00258. doi:10.1128/mBio.00258-16

26. Lokugamage KG, Hage A, Schindewolf C, Rajsbaum R, Menachery VD. SARS-CoV-2 is sensitive to type I interferon pretreatment. bioRxiv 2020. doi:10.1101/2020.03.07.982264

27. Moriguchi T, Harii N, Goto J, et al. A first case of meningitis/encephalitis associated with SARS-Coronavirus-2. Int J Infect Dis 2020; 94:55-58. doi:10.1016/j.ijid.2020.03.062

28. Baig AM. Neurological manifestations in COVID-19 caused by SARSCoV-2. CNS Neurosci Ther 2020; 26(5):499-501. doi:10.1111/cns.13372

29. To KF, Lo AWI. Exploring the pathogenesis of severe acute respiratory syndrome (SARS): the tissue distribution of the coronavirus (SARS-CoV) and its putative receptor, angiotensin-converting enzyme 2 (ACE2). J Pathol 2004; 203(3):740-743. doi:10.1002/path.1597

30. Lukassen S, Chua RL, Trefzer T, et al. SARS-CoV-2 receptor ACE2 and TMPRSS2 are primarily expressed in bronchial transient secretory cells. EMBO J 2020 Apr 14:e105114. doi:10.15252/embj.20105114

31. Hamming I, Timens W, Bulthius MLC, Lely AT, Navis GJ, van Goor $H$. Tissue distribution of ACE2 protein, the functional receptor for SARS coronavirus. A first step in understanding SARS pathogenesis. J Pathol 2004; 203(2):631-637. doi:10.1002/path.1570

32. Chen L, Li X, Chen M, Feng Y, Xiong C. The ACE2 expression in human heart indicates new potential mechanism of heart injury among patients infected with SARS-CoV-2. Cardiovasc Res 2020 Mar 30; cvaa078. doi:10.1093/cvr/cvaa078

33. Gheblawi $\mathbf{M}$, Wang $K$, Viveiros $\mathbf{A}$, et al. Angiotensin converting enzyme 2: SARS-CoV-2 receptor and regulator of the renin-angiotensin system. Circ Res 2020 Apr 8. doi:10.1161/CIRCRESAHA.120.317015

34. laccarino G, Borgi C, Cicero AFG, et al. Renin-angiotensin system inhibition in cardiovascular patients at the time of COVID19: much ado for nothing? A statement of activity from the directors of the Board and the Scientific Directors of the Italian Society of Hypertension. High Blood Press Cardiovasc Prev 2020; 27(2):105-108. doi:10.1007/s40292-020-00380-3

35. Rossi GP, Sanga V, Barton M. Potential harmful effects of discontinuing ACE-inhibitors and ARBs in COVID-19 patients. Elife 2020; 9:e57278. doi:10.7554/eLife.57278

36. Channappanavar R, Perlman S. Pathogenic human coronavirus infections: causes and consequences of cytokine storm and immuno- pathology. Semin Immunopathol 2017; 39(5):529-539. doi:10.1007/s00281-017-0629-x

37. Zhang W, Zhao Y, Zhang F, et al. The use of anti-inflammatory drugs in the treatment of people with severe coronavirus disease 2019 (COVID-19): the perspectives of clinical immunologists from China. Clin Immunol 2020; 214:108393. doi:10.1016/j.clim.2020.108393

38. Huang C, Wang Y, Li X, et al. Clinical features of patients infected with 2019 novel coronavirus in Wuhan, China. Lancet 2020; 395(10223)497-506. doi:10.1016/S0140-6736(20)30183-5

39. Wu C, Chen X, Cai Y, et al. Risk factors associated with acute respiratory distress syndrome and death in patients with coronavirus disease 2019 pneumonia in Wuhan, China. JAMA Intern Med 2020 Mar 13;e200994. doi:10.1001/jamainternmed.2020.0994

40. Ng OW, Chia A, Tan AT, et al. Memory T cell responses targeting the SARS coronavirus persist up to 11 years post-infection. Vaccine 2016 34(17):2008-2014. doi:10.1016/j.vaccine.2016.02.063

41. Peng H, Yang L-t, Wang L-y. Long-lived memory T lymphocyte responses against SARS coronavirus nucleocapsid protein in SARSrecovered patients. Virology 2006; 351(2):466-475. doi:10.1016/j.virol.2006.03.036

42. Yang LT, Peng H, Zhu Z-L, et al. Long-lived effector/central memory T-cell responses to severe acute respiratory syndrome coronavirus (SARS-CoV) S antigen in recovered SARS patients. Clin Immunol 2006; 120(2):171-178. doi:10.1016/j.clim.2006.05.002

43. To KK-W, Tsang OT-Y, Leung W-S, et al. Temporal profiles of viral load in posterior oropharyngeal saliva samples and serum antibody responses during infection by SARS-CoV-2: an observational cohort study. Lancet Infect Dis 2020 Mar 23;S1473-3099(20)30196-1. doi:10.1016/S1473-3099(20)30196-1

44. Guo L, Ren L, Yang S, et al. Profiling early humoral response to diagnose novel coronavirus disease (COVID-19). Clin Infect Dis 2020 Mar 21;ciaa310. doi:10.1093/cid/ciaa310

45. Shen C, Wang Z, Zhao F, et al. Treatment of 5 critically ill patients with COVID-19 with convalescent plasma. JAMA $2020 \mathrm{Mar}$ 27:e204783. doi:10.1001/jama.2020.4783

46. Gerna G, Campanini G, Rovida F, et al. Genetic variability of human coronavirus OC43-, 229E-, and NL63-like strains and their association with lower respiratory tract infections of hospitalized infants and immunocompromised patients. J Med Virol 2006; 78(7):938-949. doi:10.1002/jmv.20645

47. Forster P, Forster L, Renfrew C, Forster M. Phylogenetic network analysis of SARS-CoV-2 genomes. Proc Natl Acad Sci U S A 2020 Apr 8;202004999. doi:10.1073/pnas.2004999117

48. Cagliani R, Forni D, Clerici M, Sironi M. Computational inference of selection underlying the evolution of the novel coronavirus, SARS CoV-2. J Virol 2020 Apr 1;JVI.00411-20. doi:10.1128/JVI.00411-20

Addresses:

Cornelia Bergmann, PhD, Department of Neurosciences, NC30, Cleveland Clinic, 9500 Euclid Avenue, Cleveland, OH 44195; bergmac@ccf.org Robert Silverman, PhD, Department of Cancer Biology, NB40, Cleveland Clinic, 9500 Euclid Avenue, Cleveland, OH 44195; silverr@ccf.org 